\title{
Estoque de nutrientes sob diferentes sistemas de uso do solo de Colorado do Oeste-RO
}

\author{
Sulamirtes Suellem de Amorim MAGALHÁES ${ }^{1}$, Oscarlina Lúcia dos Santos WEBER ${ }^{2}$ \\ Carlos Henrique dos SANTOS ${ }^{3}$, Franciele Caroline de Assis VALADÃO ${ }^{4}$
}

RESUMO

A substituição da floresta por outro tipo de uso do solo pode levar a perdas significativas na matéria orgânica do solo, alterando sua dinâmica e como consequência alterando as entradas e saídas de nutrientes do sistema. Neste trabalho o objetivo foi avaliar o estoque de nutrientes em diferentes sistemas de uso de solo em Colorado do Oeste-RO. Os sistemas avaliados foram o agroflorestal (teca e cacau), florestal (teca com cinco anos), teca com oito anos, agrossilvopastoril (teca, cacau e pasto) e pastagem tendo a mata nativa como referência. Em cada sistema foram abertas três minitrincheiras, nas quais foram coletadas amostras deformadas e indeformadas nas profundidades de 0 a 5, 5 a 10, 10 a 20 e 20 a $30 \mathrm{~cm}$. Nessas amostras foram determinados os nutrientes: N, P, K, Ca e Mg, a matéria orgânica, a densidade do solo e os teores de argila. Observaram-se diferenças nos estoques de nutrientes no solo entre os sistemas de uso e em profundidade. A concentração de nutrientes foi influenciada pelo sistema de uso do solo. O nutriente mais estocado em todos os sistemas de uso é o Ca, os valores variaram entre 5188,48 e $2912,86 \mathrm{~kg} \mathrm{ha}^{-1}$ e o menos estocado o $\mathrm{K}$, com estoques entre 46,22 e 17,33 $\mathrm{kg} \mathrm{ha}^{-1}$. O sistema teca com cinco anos foi o sistema que mais se aproximou da mata, quanto ao armazenamento de nutrientes.

PALAVRAS-CHAVE: sistemas agroflorestais, matéria orgânica, fertilidade do solo

\section{Nutrient stocks under different land use systems of a soil in Colorado do Oeste-RO}

\begin{abstract}
The replacement of forest by other land use can lead to significant losses in soil organic matter, changing its dynamic and the nutrients inputs and outputs of the system. The objective of this study was to assess nutrient stocks under different land use systems in Colorado do Oeste-RO. The agroforestry (teak, and cocoa), forestry (teak with five years), teak for eight years, farming areas (teak, cocoa and pasture) and pasture were the systems evaluated and the native forest was taken as reference. In each system three small trenches were dug, in which deformed and undeformed soil samples, at depths of 0 to 5, 5 to 10,10 to 20 and 20 to $30 \mathrm{~cm}$ were taken. In these samples the nutrients $\mathrm{N}, \mathrm{P}, \mathrm{K}, \mathrm{Ca}$ and $\mathrm{Mg}$, organic matter, bulk density and clay content were determined. A completely randomized design with split plots and, three replications in each system was adopted. Differences were observed in soil nutrient stocks among the usage systems and with depth. The concentration of nutrients was influenced by the system. Ca was the nutrient most stored (2912.86 to $\left.5188.48 \mathrm{~kg} \mathrm{ha}^{-1}\right)$ in all systems and $\mathrm{K}$ was the least $\left(17.33\right.$ to $\left.46.22 \mathrm{~kg} \mathrm{ha}^{-1}\right)$. The system of teak five years is the system which is closer to the forest, for the storage of nutrients.
\end{abstract}

KEYWORDS: agroforestry, organic matter, soil fertility

\footnotetext{
1 Programa de Pós-Graduação em Agricultura Tropical, Universidade Federal de Mato Grosso- Faculdade de Agronomia e Medicina Veterinária UFMT-FAMEV, Av. Fernando Corrêa, Cuiabá, 78060900,sulamorim@yahoo.com.br ;

2 Programa de Pós-Graduação em Agricultura Tropical, Universidade Federal de Mato Grosso- Faculdade de Agronomia e Medicina Veterinária UFMT/FAMEV. Av Fernando Corrêa Cuiabá, 78060900, oscsanwb@ufmt.br:

3 Instituto Federal de Rondônia IFR0- campus Colorado do Oeste, End, Colorado do Oeste-R0, CEP 76993-000, carlos.henrique@ifro.edu.br ;

${ }^{4}$ Programa de Pós-Graduação em Agricultura Tropical, Universidade Federal de mato Grosso- Faculdade de Agronomia e Medicina Veterinária UFMT/FAMEV, Av. Fernando Corrêa, Cuiabá, 78060900, agroassis@gmail.com ;
} 


\section{INTRODUÇÃO}

A agricultura itinerante, principal meio de cultivo das terras na Amazônia, pode promover várias modificaçôes nas propriedades físicas, químicas e biológicas destas, a partir do momento em que o sistema natural é modificado.

Inicialmente, após o desmatamento, a produçáo das culturas tende a ser alta devido à incorporação das cinzas provenientes da queima da floresta. Com o decorrer do tempo de cultivo, devido ao consumo contínuo de nutrientes pelas culturas, a produtividade cai em função da redução dos níveis de fertilidade. Esse efeito é mais intenso quando não se utilizam práticas corretivas e fertilização do solo (Moreira e Costa 2004).

Uma alternativa para manter ou melhorar a fertilidade, é a utilizaçấo de sistemas de culturas que possam adicionar material orgânico, proporcionando um balanço entre a adiçáo e a retirada ou perda desses elementos por meio dos sistemas de cultivo, visto que os estoques de matéria orgânica em qualquer agroecossistema são obtidos pela interação dos fatores que determinam sua formação e aqueles que promovem sua decomposição (Altieri 2002).

A implantação de sistemas agroflorestais (SAFs) tem emergido como alternativa de uso da terra para pequenos agricultores da América Tropical, devido ao seu potencial de reduzir a degradação do solo e diminuir a pressão sobre as áreas de florestas (Mcgrath et al. 2001).

Os SAFs caracterizam-se pela combinaçáo de espécies arbóreas com culturas agrícolas e, ou, com animais domésticos, simultaneamente, ou alternados no tempo e no espaço. $\mathrm{Na}$ Amazônia, o grande desafio tem sido desenvolver sistemas capazes de conciliar, de forma harmoniosa, interesses de conservaçáo ambiental com sustentabilidade econômica, em substituição à agricultura migratória comumente praticada (Galvão et al. 2009).

Em sistemas agrossilvopastoris e agroflorestais há poucas informaçôes sobre a capacidade desses em estocar nutrientes no solo. Os SAFs são uma das formas de uso que menos alteram as propriedades químicas, físicas e a distribuição da matéria orgânica no solo, além de promover mais aporte de carbono ao solo pela fração orgânica leve (Costa 2004).

Dessa forma, partindo da hipótese que a mudança nos diferentes sistemas de uso do solo altera a dinâmica da matéria orgânica e o estoque de nutrientes do solo, o objetivo deste trabalho foi avaliar o estoque de nutrientes de solo no município de Colorado do Oeste, em Rondônia sob diferentes sistemas de uso.

\section{MATERIAL E MÉTODOS}

O experimento foi desenvolvido no sítio Estrela, linha 2, $\mathrm{km} \mathrm{9,5}$ rumo ao Rio Escondido, em uma área pertencente ao município de Colorado do Oeste, regiáo sul do estado de Rondônia, coordenadas geográficas 1309'29,4” S e 60³7’50,6” W, a 333 metros de altitude.

O clima da região, de acordo com a classificação de Köppen, é do tipo AMWI, quente e úmido, apresentando duas estaçốes climáticas bem definidas: o veráo de maio a setembro, quando se observam as mais baixas precipitaçôes pluviométricas, em torno de 750 a $810 \mathrm{~mm}$, ou seja, 30 a $40 \%$ do total; e o inverno de outubro a abril, quando ocorrem as maiores precipitaçóes entre 1.470 e $1.500 \mathrm{~mm}$ ou $60 \%$ do total, ocorrendo chuvas diárias num período de 100 dias (Governo do Estado de Rondônia/SEPLAN 2002).

O relevo de Colorado do Oeste-RO varia de ondulado a montanhoso, porquanto a maior parte do município está situada na calha sudoeste da encosta da Chapada dos Parecis em altitudes maiores que $400 \mathrm{~m}$ acima do nível do mar. Os solos predominantes são do tipo Argissolos VermelhoAmarelos, Argissolos Vermelhos e Cambissolos eutróficos, sendo que o solo das áreas do estudo é um Argissolo Vermelho Amarelo Eutrófico, o material de origem é autóctone e de rocha básica metamórfica (Governo do Estado de Rondônia/ SEDAM 2010).

As áreas de estudo, consistiram de uma referência (vegetação nativa/floresta) e de cinco diferentes sistemas de uso.

Para este estudo, foram escolhidas seis áreas contíguas: (i) sob cobertura vegetal de floresta primária/mata nativa (MA); (ii) sistema agroflorestal sob teca (Tectona grandis L. F.) com oito anos (T8), (iii) sistema agroflorestal com teca com cinco anos (T5); (iv) sistema agroflorestal sob teca $\mathrm{x}$ cacau (Theobroma cacao) x pastagem (Brachiaria brizantha) com oito anos (TCP); (v) sistema agroflorestal com teca x cacau com oito anos (TC) e (vi) pastagem extensiva com braquiária (Brachiaria brizantha) com cinco anos (PA).

Os sistemas em estudo não receberam calagem nem adubação de correçáo do solo; o histórico da área pode ser verificado na Tabela 1e os atributos químicos e a granulometria estão na Tabela 2.

A amostragem foi feita em quatro profundidades, de 0 a 5 , 5 a 10,10 a 20,20 a $30 \mathrm{~cm}$ de profundidade. Foram abertas três minitrincheiras em cada área para coleta de amostras indeformadas e deformadas. 
Tabela 1 - Características dos sistemas de uso das áreas escolhidas em Colorado do 0este-Ro

\begin{tabular}{|c|c|c|}
\hline Sistema de uso & Símbolo & Histórico \\
\hline Mata nativa & MA & $\begin{array}{l}\text { Área sob floresta tropical aberta semidecídua, submontana, dossel emergente, tendo como percentual de } \\
\text { árvores caducifólias, em torno de } 20 \text { a 50\% nos períodos de seca. }\end{array}$ \\
\hline $\begin{array}{l}\text { Sistema agroflorestal com } \\
\text { teca com cinco anos }\end{array}$ & T5 & $\begin{array}{l}\text { Teca implantada em dezembro de } 2001 \text {, em } 7,0 \text { ha com espaçamento de } 3 \times 2 \mathrm{~m} \text {, onde a floresta primária } \\
\text { foi derrubada manualmente, seguida de queimada. Nos dois primeiros anos foram cultivados arroz, milho e } \\
\text { feijão consorciados entre as linhas, em seguida foi feito a cobertura do solo com kudzu (Pueraria montana) }\end{array}$ \\
\hline $\begin{array}{l}\text { Sistema agroflorestal sob } \\
\text { teca com oito anos }\end{array}$ & T8 & $\begin{array}{l}\text { Teca implantada em 1998, onde a floresta primária foi derrubada manualmente em junho de } 1975 \text {, seguida de } \\
\text { queimada, sendo plantado café (coffea canephora). Entre as linhas foi consorciado com arroz, milho e feijão } \\
\text { por } 4 \text { anos sem adubação. No quinto ano de cultivo foi introduzida pastagem de capim braquiária (Brachiaria } \\
\text { brizantha), em 1984, nove anos depois foi plantado pinho cuiabano (Shizolobium amazonicum), em } 10 \text { ha } \\
\text { com o espaçamento de } 3 \times 5 \text { m e, como algumas mudas morreram, foi plantado teca para substituí-las e em } \\
\text { dezembro de } 2000 \text { foi feita a cobertura do solo com kudzu. }\end{array}$ \\
\hline $\begin{array}{l}\text { Sistema agroflorestal sob } \\
\text { teca } \mathrm{x} \text { cacau } \mathrm{x} \text { pasto }\end{array}$ & TCP & $\begin{array}{l}\text { Teca implantada em 1998, sendo o histórico e área o mesmo descrito no sistema T8, mas em dezembro de } \\
\text { 2000, uma parte dessa área, entre as árvores nas linhas foi consorciada cacau, permanecendo nessa área a } \\
\text { cobertura com pastagem (Brachiaria brizantha) }\end{array}$ \\
\hline $\begin{array}{l}\text { Sistema agroflorestal com } \\
\text { teca } x \text { cacau com } 8 \text { anos }\end{array}$ & TC & $\begin{array}{l}\text { A mesma área e histórico do sistema T8, sendo que em dezembro de } 2000 \text { foi consorciado com cacau entre } \\
\text { as árvores na linhas. }\end{array}$ \\
\hline $\begin{array}{l}\text { Pastagem extensiva com } \\
\text { braquiária com cinco anos }\end{array}$ & PA & $\begin{array}{l}\text { Área aberta em 2001, com derrubada manual da floresta, seguida da queimada e semeadura da braquiária } \\
\text { (Brachiaria brizantha), o qual foi pastejado extensivamente pelo gado, resultando numa desuniformidade na } \\
\text { utilização do capim, ficando algumas áreas subutilizadas e outras com excessivo pastejo. }\end{array}$ \\
\hline
\end{tabular}

Para a caracterização dos atributos químicos, as amostras de solo foram secas ao ar livre, destorroadas e peneiradas em tamiz de malha de 2,00 mm, para em seguida serem analisadas. Os nutrientes; potássio, fósforo, cálcio, magnésio, a densidade do solo e o teor de argila foram analisados seguindo os métodos descritos em Embrapa (1997). A determinação da matéria orgânica total (MOT) do solo seguiu o método proposto por Conceição et al. (1999), e para o nitrogênio total foi utilizado o método descrito por Bremner e Mulvaney (1982).

$\mathrm{O}$ estoque de nutrientes nas respectivas camadas de solo foi transformado pelos métodos da camada (Bayer et al. 2000) e da massa de solo equivalentes (Ellert e Bettany 1995). Os dados obtidos foram submetidos à análise de variância e teste de médias (Scott-Knott a 5\% de probabilidade) e como análise complementar foi realizada a análise de componentes principais para verificar a relação entre as variáveis (Johnson e Wichern 1998) e a análise de agrupamento, sendo usado como medida de similaridade à distância Euclidiana e o algoritmo de agrupamento pelo Método de Ligação Completa, onde os membros são combinados pela menor distância máxima entre eles (Hair Jr. et al. 2005).

\section{RESULTADOS E DISCUSSÃO}

$\mathrm{Na}$ Tabela 3 são apresentados os resultados de estoque de nutrientes e matéria orgânica do solo, para a interação sistema de uso e profundidade do solo. Todos os sistemas de uso apresentaram maiores teores de matéria orgânica na camada superficial de 0 a $5 \mathrm{~cm}$, decrescendo em profundidade,

Tabela 2 - Médias dos atributos químicos e granulometria em diferentes sistemas de uso na profundidade de $0-30 \mathrm{~cm}$

\begin{tabular}{|c|c|c|c|c|c|c|c|c|c|c|}
\hline \multirow[b]{2}{*}{ Sistema de uso } & \multirow[b]{2}{*}{$\mathrm{pH}$} & \multicolumn{4}{|c|}{ Atributos químicos } & \multicolumn{5}{|c|}{ Granulometria } \\
\hline & & $\mathrm{pH}$ & $\mathrm{Ca}^{2+}$ & $\mathrm{Mg}^{2+}$ & $\mathrm{Al}+\mathrm{H}$ & $\mathrm{K}^{+}$ & $P$ & Areia & Silti & Argila \\
\hline & $\mathrm{H}_{2} \mathrm{O}$ & $\mathrm{CaCl}_{2}$ & \multicolumn{3}{|c|}{ cmolc dm-3 } & \multicolumn{2}{|c|}{$\mathrm{mg} \mathrm{dm}{ }^{-3}$} & \multicolumn{3}{|c|}{$\mathrm{g} \mathrm{kg}^{-1}$} \\
\hline MA & 5,9 & 5,53 & 8,9 & 2,06 & 2,14 & 11,16 & 26,78 & 528 & 164 & 308,8 \\
\hline T8 & 5,51 & 4,98 & 5,04 & 6,38 & 2,88 & 9 & 48,16 & 680 & 90 & 244,31 \\
\hline $\mathrm{T} 5$ & 6,57 & 6,08 & 7,45 & 2,07 & 1,7 & 10,77 & 150,85 & 666 & 122 & 326,71 \\
\hline $\mathrm{TCP}$ & 5,76 & 5,31 & 4,06 & 4,71 & 2,6 & 5,18 & 14,57 & 593 & 154 & 282,13 \\
\hline $\mathrm{TC}$ & 5,93 & 5,42 & 5,48 & 3,85 & 2,94 & 5,58 & 39,71 & 654 & 120 & 238,8 \\
\hline PA & 5,94 & 5,75 & 6,42 & 1,93 & 1,73 & 12,63 & 17,84 & 405 & 248 & 372,33 \\
\hline
\end{tabular}


Tabela 3 - Estoque de nutrientes e teores de matéria orgânica em relação aos sistemas de uso e profundidade do solo

\begin{tabular}{|c|c|c|c|c|c|c|}
\hline Prof (cm) & $\mathrm{MA}^{1}$ & $\mathrm{~T}^{2}$ & $T 5^{3}$ & $\mathrm{TCP}^{4}$ & $\mathrm{TC}^{5}$ & $\mathrm{PA}^{6}$ \\
\hline \multicolumn{7}{|c|}{ NT kg ha-1 } \\
\hline 0 a 5 & $2068,37 \mathrm{Aa}$ & $1014,44 \mathrm{Da}$ & $1203,95 \mathrm{Ca}$ & $869,52 \mathrm{Ea}$ & $819,36 \mathrm{Ea}$ & $1538,39 \mathrm{Ba}$ \\
\hline 5 a 10 & $728,85 \mathrm{Bb}$ & $677,27 \mathrm{Bb}$ & $920,40 A b$ & $602,02 \mathrm{Bb}$ & $399,42 \mathrm{Cb}$ & $364,69 \mathrm{Cd}$ \\
\hline 10 a 20 & $875,61 \mathrm{Bb}$ & $1094,52 \mathrm{Aa}$ & $1240,46 \mathrm{Aa}$ & $1021,55 \mathrm{Aa}$ & $681,03 \mathrm{Ca}$ & $1058,03 \mathrm{Ab}$ \\
\hline 20 a 30 & $775,38 \mathrm{Ab}$ & $827,64 \mathrm{Ab}$ & $882,82 A b$ & $869,02 \mathrm{Aa}$ & $744,88 \mathrm{Aa}$ & $993,17 \mathrm{Ac}$ \\
\hline \multicolumn{7}{|c|}{$P$ kg ha-1 } \\
\hline 0 a 5 & $22,48 \mathrm{Da}$ & $54,83 \mathrm{Ba}$ & $115,69 \mathrm{Ac}$ & $10,80 \mathrm{Da}$ & $41,00 \mathrm{Cb}$ & $14,34 \mathrm{Da}$ \\
\hline 5 a 10 & $19,02 \mathrm{Ca}$ & $35,48 \mathrm{Bb}$ & $99,53 \mathrm{Ad}$ & $9,40 \mathrm{Ca}$ & $25,13 \mathrm{Ba}$ & $10,74 \mathrm{Ca}$ \\
\hline 10 a 20 & $16,20 \mathrm{Ba}$ & $22,18 \mathrm{Bb}$ & $164,69 \mathrm{Ab}$ & 13,67Ba & $12,99 \mathrm{Ba}$ & $21,23 \mathrm{Ba}$ \\
\hline 20 a 30 & $29,75 \mathrm{Ba}$ & $23,96 \mathrm{Bb}$ & $202,13 \mathrm{Aa}$ & $9,14 \mathrm{Ca}$ & $18,08 \mathrm{Ca}$ & $17,13 \mathrm{Ca}$ \\
\hline \multicolumn{7}{|c|}{$\mathrm{K} \mathrm{kg} \mathrm{ha}^{-1}$} \\
\hline 0 a 5 & $8,03 \mathrm{Bb}$ & $6,83 \mathrm{BC}$ & $7,46 \mathrm{Bb}$ & $4,13 \mathrm{Cb}$ & $4,13 \mathrm{Ca}$ & $10,35 \mathrm{Ab}$ \\
\hline 5 a 10 & $6,42 \mathrm{Ac}$ & $4,62 \mathrm{Bd}$ & $5,19 \mathrm{BC}$ & $2,97 \mathrm{Cb}$ & $3,21 \mathrm{Ca}$ & $7,92 \mathrm{Ac}$ \\
\hline 10 a 20 & $12,51 \mathrm{Aa}$ & $9,35 \mathrm{Bb}$ & $10,39 \mathrm{Ba}$ & $4,68 \mathrm{Cb}$ & $4,68 \mathrm{Ca}$ & $9,87 \mathrm{Bb}$ \\
\hline 20 a 30 & 13,07Ba & $11,20 \mathrm{Ca}$ & $10,02 \mathrm{Ca}$ & $14,15 \mathrm{Ba}$ & $5,31 \mathrm{Da}$ & $18,08 \mathrm{Aa}$ \\
\hline \multicolumn{7}{|c|}{ Ca kg ha-1 } \\
\hline 0 a 5 & $1734,36 \mathrm{Aa}$ & $973,34 \mathrm{Ba}$ & $1148,86 \mathrm{Bb}$ & $506,62 \mathrm{Db}$ & $961,37 \mathrm{Ba}$ & $698,09 \mathrm{Cb}$ \\
\hline 5 a 10 & $779,64 \mathrm{Ad}$ & $580,00 \mathrm{Bb}$ & $857,57 \mathrm{Ac}$ & $596,57 \mathrm{Bb}$ & $596,57 \mathrm{Bb}$ & $836,85 \mathrm{Ab}$ \\
\hline 10 a 20 & $1488,76 \mathrm{Ab}$ & $609,25 \mathrm{Cb}$ & $1523,13 \mathrm{Aa}$ & $983,51 \mathrm{Ba}$ & $939,99 \mathrm{Ba}$ & $1497,02 \mathrm{Aa}$ \\
\hline 20 a 30 & $1185,72 \mathrm{Bc}$ & $750,28 \mathrm{Cb}$ & $1046,44 \mathrm{Bb}$ & $1125,42 \mathrm{Ba}$ & $977,34 \mathrm{Ba}$ & $1540,05 \mathrm{Aa}$ \\
\hline \multicolumn{7}{|c|}{$\mathrm{Mg} \mathrm{kg} \mathrm{ha}^{-1}$} \\
\hline 0 a 5 & $144,96 \mathrm{Bb}$ & $181,44 \mathrm{Bb}$ & $94,35 \mathrm{Ba}$ & $319,34 \mathrm{Ac}$ & $321,76 \mathrm{Aa}$ & $135,48 \mathrm{Ba}$ \\
\hline 5 a 10 & $169,11 \mathrm{Cb}$ & $711,04 \mathrm{Aa}$ & $160,80 \mathrm{Ca}$ & $424,61 \mathrm{Bc}$ & $173,36 \mathrm{Ca}$ & $251,25 \mathrm{Ca}$ \\
\hline 10 a 20 & $355,33 \mathrm{Ba}$ & $702,04 \mathrm{Aa}$ & $216,42 \mathrm{Ba}$ & $649,25 \mathrm{Ab}$ & $131,96 \mathrm{Ba}$ & $206,39 \mathrm{Ba}$ \\
\hline 20 a 30 & $288,88 \mathrm{Ba}$ & $239,49 \mathrm{Bb}$ & $125,73 \mathrm{Ba}$ & $1077,68 \mathrm{Aa}$ & $185,60 \mathrm{Ba}$ & $317,32 \mathrm{Ba}$ \\
\hline \multicolumn{7}{|c|}{$\mathrm{MO} \mathrm{g} \mathrm{kg}^{-1}$} \\
\hline 0 a 5 & $84,50 \mathrm{Aa}$ & $47,02 \mathrm{Ca}$ & $55,95 \mathrm{Ba}$ & $59,51 \mathrm{Ba}$ & $35,97 \mathrm{Da}$ & $61,96 \mathrm{Ba}$ \\
\hline 5 a 10 & $28,08 \mathrm{Ab}$ & $19,36 \mathrm{Bb}$ & $32,58 \mathrm{Ab}$ & $24,50 \mathrm{Bb}$ & $22,12 \mathrm{Bb}$ & $24,36 \mathrm{Bb}$ \\
\hline 10 a 20 & $12,93 \mathrm{Ac}$ & $9,54 \mathrm{Ac}$ & $14,47 \mathrm{Ac}$ & $15,06 \mathrm{Ac}$ & $12,12 \mathrm{Ac}$ & $19,90 \mathrm{Ab}$ \\
\hline 20 a 30 & $10,84 \mathrm{Bc}$ & $5,71 \mathrm{Bc}$ & $8,53 \mathrm{BC}$ & $11,62 \mathrm{Bc}$ & $12,12 \mathrm{BC}$ & $23,57 \mathrm{Ab}$ \\
\hline
\end{tabular}

$M A^{1}=$ mata, $T 8^{2}$ teca 8 anos, T5³ =teca 5anos, TCP4 =teca, cacau e pasto, TC ${ }^{5}=$ teca e cacau, PA6=pasto ,NT (Nitrogênio total), P (fósforo), K (potássio), Ca (Cálcio), Mg (Magnésio), e M0 (Matéria orgânica). Médias seguidas de letras diferentes, maiúscula na horizontal e minúscula na vertical, diferem entre si pelo teste Scott-Knott $(P<0,05)$.

o que deve-se provavelmente ao maior aporte de serrapilheira (fragmentos de folhas, galhos e raízes) que promove maiores modificações nessa camada (Rangel e Silva 2007).

O sistema MA apresentou os maiores teores de matéria orgânica nas camadas estudadas, com exceção da camada de
20 a $30 \mathrm{~cm}$, onde o PA foi o que apresentou o maior teor de matéria orgânica, sendo este valor $54 \%$ maior que no sistema MA. Como no sistema PA houve queima da vegetação para sua implantação, os maiores teores de matéria orgânica na 
camada de 20 a $30 \mathrm{~cm}$ podem ser decorrentes da incorporação das cinzas (Araújo et al. 2011).

Os maiores estoques de nitrogênio total (NT) foram verificados nos sistemas MA e T5, o sistema MA foi o que mais armazenou esse nutriente na camada de 0 a $5 \mathrm{~cm}$, o valor foi de $2068,37 \mathrm{~kg} \mathrm{ha}^{-1}$, nas outras profundidades o sistema T5 foi o que mais contribuiu no armazenamento de NT com valores entre 1240,46 e 882,82 $\mathrm{kg} \mathrm{ha}^{-1}$. Esses resultados estão associados ao maior volume de resíduos vegetais retornados ao solo e, também, aos maiores teores de matéria orgânica nesses sistemas (Rangel e Silva 2007).

Nos sistemas MA e PA, o NT decresceu em profundidade, diferenciando-se dos sistemas agroflorestais onde houve aumento de NT em profundidade. A maior parte do NT contido no solo está associado à matéria orgânica, justificando seu maior acúmulo na camada superficial, nos sistemas MA e PA. A mineralização da matéria orgânica resulta não só na acumulação de NT, como também de P, K, Ca e Mg (Moreira e Malavolta 2004), consequentemente, a estabilidade da matéria orgânica é importante na reserva de nutrientes dos solos.

Com relaçáo ao estoque de $\mathrm{P}$, houve aumento em profundidade apenas nos sistemas T5 e TC. Os sistemas TCP e PA foram os que menos estocaram esse nutriente. O P é um nutriente importante no estabelecimento e produtividade das pastagens, sendo essencial para o crescimento radicular, perfilhamento e produçáo de biomassa (Silva et al. 2007). Desse modo, baixo estoque de $\mathrm{P}$ na área de pastagem poderá influenciar a produtividade, indicando que é necessário além do manejo adequado, a entrada do nutriente via fertilização para a manutenção da produtividade inicial ou acréscimo da mesma, do contrário, em pouco tempo a produtividade diminuirá e o agricultor abandonará a área, derrubando novas parcelas de mata nativa (Araújo et al. 2004).

Assim como o nitrogênio, o cálcio também foi armazenado em grande quantidade em todos os sistemas estudados, o armazenamento foi entre $1734,36 \mathrm{e} 506,62 \mathrm{~kg} \mathrm{ha}^{-1}$, podendo sugerir que o material desse solo é rico nesse nutriente e pobre em outros nutrientes como o K. Na camada de 0 a $5 \mathrm{~cm}$ o sistema MA foi que o mais armazenou $\mathrm{Ca}\left(1734,36 \mathrm{~kg} \mathrm{ha}^{-1}\right)$, sendo que nas camadas adjacentes além da MA, os sistemas T5 e PA também se destacaram no armazenamento de Ca.

Nos sistemas T5, TCP e TC e PA houve um aumento de $\mathrm{Ca}$ em profundidade. Também foi verificado, aumento em profundidade para os nutrientes $\mathrm{Mg}$ e K.

Apesar dos sistemas terem acumulado maior quantidade de nutrientes em profundidade isso não reverteu em maior nível de matéria orgânica no solo em profundidade, pois como já foi comentado, sua quantidade foi maior apenas na camada de 0 a $5 \mathrm{~cm}$, decrescendo nas demais profundidades (Tabela 3).

Dentre os fatores que podem ter contribuído para o aumento do estoque de nutrientes no perfil, destacam-se a preservaçáo das características físicas do solo nos sistemas de uso estudados devido à ausência de revolvimento do solo e a provável existência de canais contínuos, oriundos da decomposição de raízes e da atividade biológica (Mokry 2003), possibilitando o aumento do estoque de nutrientes em profundidade devido à translocaçấo de nutrientes da camada superficial do solo.

$\mathrm{O}$ estoque total de nutrientes no perfil do solo (camada de 0 a $30 \mathrm{~cm}$ ) seguiu as sequências; no sistema $\mathrm{MA} \quad \mathrm{Ca}>\mathrm{NT}>\mathrm{Mg}>\mathrm{P}=\mathrm{K}$; T $8 \quad \mathrm{NT}>\mathrm{Ca}>\mathrm{Mg}>\mathrm{P}=\mathrm{K}, \mathrm{T} 5$ $\mathrm{NT}=\mathrm{Ca}>\mathrm{Mg}=\mathrm{P}>\mathrm{K}$, no sistema TCP $\mathrm{N}=\mathrm{Ca}>\mathrm{Mg}>\mathrm{P}=\mathrm{K}$, no TC $\mathrm{Ca}>\mathrm{NT}>\mathrm{Mg}>\mathrm{P}=\mathrm{K}$ e no $\mathrm{PA} \mathrm{Ca}>\mathrm{NT}>\mathrm{Mg}>\mathrm{P}=\mathrm{K}$ (Tabela 4). Verificou-se que, de modo geral, o Ca e o NT foram os nutrientes mais estocados, sendo o Ca mais estocado nos sistemas MA, TC e PA e o NT mais estocado apenas no sistema T8.

No sistema MA os estoques de Ca estiveram acima de $600 \mathrm{~kg} \mathrm{ha}^{-1}$, semelhante aos encontrados em solos de floresta primária por Araújo et al. (1994). Os sistemas MA, T5 e PA foram os que mais armazenaram Ca e NT. Uma das prováveis causas, que podem ter contribuído para o aumento do armazenamento de $\mathrm{Ca}$, é a decomposição do sistema radicular da mata primitiva, que promoveu a liberação desse nutriente, ou ainda, no caso dos sistemas de uso, os altos teores de cálcio podem ser explicados pela queima da mata na implantaçáo de outros sistemas, sendo atribuído à concentração desse elemento nas cinzas (Araújo et al. 2004), já que os sistemas

Tabela 4 - Estoque total de nutrientes na camada de 0 a $30 \mathrm{~cm}$ dentro de cada sistema de uso

\begin{tabular}{|c|c|c|c|c|c|c|c|}
\hline Estoque & $\mathrm{kg} \mathrm{ha}^{-1}$ & $\mathrm{MA}^{1}$ & $T 8^{2}$ & $\mathrm{~T} 5^{3}$ & $\mathrm{TCP}^{4}$ & $\mathrm{TC}^{5}$ & $\mathrm{PA}^{6}$ \\
\hline NT & & $4448,21 \mathrm{~b}$ & $3613,88 \mathrm{a}$ & $4357,98 \mathrm{a}$ & $3375,92 \mathrm{a}$ & $2644,69 \mathrm{~b}$ & $3830,13 b$ \\
\hline$P$ & & $87,45 d$ & $136,45 d$ & $582,03 \mathrm{~b}$ & $43,01 \mathrm{c}$ & $97,21 d$ & $63,44 \mathrm{~d}$ \\
\hline K & & $40,02 d$ & $32,00 \mathrm{~d}$ & $33,07 \mathrm{c}$ & $25,92 \mathrm{c}$ & $17,33 d$ & $46,22 d$ \\
\hline $\mathrm{Ca}$ & & $5188,48 \mathrm{a}$ & 2912,86 b & $4576,00 \mathrm{a}$ & $3212,11 \mathrm{a}$ & $3475,27 \mathrm{a}$ & $4572,01 \mathrm{a}$ \\
\hline $\mathrm{Mg}$ & & $958,28 \mathrm{c}$ & $1834,00 \mathrm{c}$ & $597,29 b$ & $2470,88 b$ & $812,68 \mathrm{c}$ & $910,43 \mathrm{c}$ \\
\hline
\end{tabular}

$\mathrm{MA}^{1}=$ mata, $\mathrm{T}^{2}$ teca 8 anos, $\mathrm{T}^{3}=$ teca 5 anos, TCP $=$ teca, cacau e pasto, $\mathrm{TC}^{5}=$ teca e cacau, $\mathrm{PA}{ }^{6}=$ pasto.NT (Nitrogênio total), $\mathrm{P}$ (fósforo), $\mathrm{K}$ (potássio), $\mathrm{Ca}$ (Cálcio) e Mg (Magnésio). Médias seguidas de letras diferentes, minúscula na vertical, diferem entre si pelo teste Scott-Knott $(P<0,05)$. 
em estudo náo receberam calagem e nem adubação de correçáo do solo.

Essa manutenção dos estoques de NT que o sistema T8 proporcionou, tendo o sistema MA como referência, demonstrou que a adoção desse modelo de cultivo resultou em maior aporte desse elemento ao solo, cerca de $3610 \mathrm{~kg}$ ha $^{-1}$, concordando com Silva et al. (2004).

O P foi um dos nutrientes menos estocado nos sistemas de uso estudados, o armazenamento foi entre 136,45 e 43,01 $\mathrm{kg} \mathrm{ha}^{-1}$, indicando que o mesmo foi alterado pelo processo de derrubada e queima, sendo reduzido, não atingindo o equilíbrio no solo (Araújo et al. 2004)

Observou-se que o nutriente mais limitante nos sistemas de uso é o K. Neste estudo foram encontrados valores de $40,02 \mathrm{~kg} \mathrm{ha}^{-1}$ na MA, abaixo do encontrado por Silva (1995), que encontrou em solos de floresta primária estoque de $\mathrm{K} \mathrm{em}$ torno de $66 \mathrm{~kg} \mathrm{ha}^{-1}$.

Assim o estoque de $\mathrm{K}$ não se assemelhou ao cálcio, monstrando a possibilidade de ocorrer perdas por lixiviação, o que está relacionado ao grau de intemperismo dos solos (Marques et al. 2010). Outra possível causa do baixo estoque de $\mathrm{K}$, pode ser devido à interação eletrostática entre $\mathrm{Ca}, \mathrm{Mg}$ e K (Swarowsky et al. 2006). Os cátions bivalentes (Ca e $\mathrm{Mg}$ ) estáo adsorvido mais fortemente aos colóides do solo. Assim, excesso de Ca e/ou Mg no solo geralmente dificultam o armazenamento do K (Ernani 2008).

$\mathrm{O} \mathrm{Mg}$ foi o terceiro nutriente mais estocado em todos os sistemas de uso. Apesar desse nutriente apresentar padráo similar ao Ca no solo, pois é adsorvido aos colóides do solo (Ernani 2008), ele não foi armazenado em grande quantidade como o Ca nos sistemas de uso.
$\mathrm{O}$ aumento dos estoques de nutrientes como $\mathrm{P}$ e $\mathrm{Mg}$ nos sistemas T5 e TCP, quando comparado ao MA está relacionado à melhoria da qualidade do solo, proporcionado pelas interaçóes entre os componentes florestal e agrícola que resultam no maior aporte de fitomassa no solo. Além disso, a ausência de revolvimento do solo desse sistema proporciona melhores condiçóes para os organismos responsáveis pela fragmentação do material vegetal e ciclagem dos nutrientes (Lima et al. 2011).

Com relação à densidade (Tabela 5), nos sistemas MA, T5 e PA os valores foram de 1,30, 1,38 e $1,23 \mathrm{Mg} \mathrm{m}^{-3}$ respectivamente, sendo menores do que encontrados nos sistemas T8, TCP e TC. Essas três últimas áreas estavam anteriormente ocupadas com pastagem conforme histórico (Tabela 1), de modo que o pisoteio no solo sob pastagem pelo gado, pode ter contribuído para maior densidade nesses sistemas (Martinez e Zinck 2004).

No PA a densidade média foi de $1,23 \mathrm{Mg} \mathrm{m}^{-3}$; valores médios de densidade sob pastagem foram obtidos por Souza e Alves (2003) na ordem de $1,56 \mathrm{Mg} \mathrm{m}^{-3}$ e $1,54 \mathrm{Mg} \mathrm{m}^{-3}$, na profundidade de $0-20 \mathrm{~cm}$ do solo. Este resultado da densidade no sistema de pastagem pode ser explicado devido ao curto tempo de estabelecimento da pastagem, para mudar as características físicas do solo (Longo e Espíndola 2000). Falleiro et al. (2003) observaram diferença na densidade do solo depois de 16 anos consecutivos de diferentes sistemas de preparo.

Assim como o sistema PA, o solo sob MA apresentou menor valor de densidade, o que se deve principalmente pela maior quantidade de matéria orgânica nesse sistema. Verificouse que a densidade aumentou em profundidade nos sistemas

Tabela 5 - Densidade e teor de argila em diferentes sistemas de uso do solo

\begin{tabular}{|c|c|c|c|c|c|c|c|}
\hline Profun (cm) & $\mathrm{MA}^{1}$ & $T 8^{2}$ & $\mathrm{~T}^{3}$ & $\mathrm{TCP}^{4}$ & $\mathrm{TC}^{5}$ & $\mathrm{PA}^{6}$ & Média \\
\hline \multicolumn{8}{|c|}{ Densidade $\mathrm{Mg} \mathrm{m}^{3}$} \\
\hline 0 a 5 & 1,19 & 1,43 & 1,26 & 1,41 & 1,47 & 1,07 & $1,30 \mathrm{c}$ \\
\hline 5 a 10 & 1,24 & 1,47 & 1,33 & 1,45 & 1,48 & 1,21 & $1,36 \mathrm{c}$ \\
\hline 10 a 20 & 1,30 & 1,49 & 1,47 & 1,54 & 1,57 & 1,27 & $1,44 b$ \\
\hline 20 a 30 & 1,47 & 1,63 & 1,46 & 1,62 & 1,66 & 1,39 & $1,54 \mathrm{a}$ \\
\hline Média & $1,30 \mathrm{~B}$ & $1,51 \mathrm{~A}$ & $1,38 \mathrm{~B}$ & $1,50 \mathrm{~A}$ & $1,54 \mathrm{~A}$ & $1,23 \mathrm{~B}$ & \\
\hline \multicolumn{8}{|l|}{ Argila $\mathrm{g} \mathrm{kg}^{-1}$} \\
\hline 0 a 5 & 302,13 & 255,46 & 308,46 & 368,80 & 248,80 & 390,66 & $312,38^{\text {ns }}$ \\
\hline 5 a 10 & 315,46 & 255,46 & 332,80 & 248,80 & 235,46 & 344,00 & 288,66 \\
\hline 10 a 20 & 308,80 & 184,20 & 332,80 & 242,13 & 235,46 & 344,00 & 274,56 \\
\hline 20 a 30 & 308,80 & 282,13 & 332,80 & 268,80 & 235,46 & 410,66 & 306,44 \\
\hline Média & $308,80 \mathrm{~A}$ & 244,31 B & $326,71 \mathrm{~A}$ & 282,13 B & $238,80 \mathrm{~B}$ & $372,33 \mathrm{~A}$ & 295,51 \\
\hline
\end{tabular}

$\mathrm{MA}^{1}=$ mata, $\mathrm{T}^{2}$ teca 8 anos, $\mathrm{T}^{3}=$ teca 5 anos, $\mathrm{TCP}{ }^{4}=$ teca, cacau e pasto, $\mathrm{TC}^{5}=$ teca e cacau, $\mathrm{PA}^{6}=$ pasto.ns, não significativo pelo teste de Scott-Knott $(\mathrm{P}<0,05)$. Médias seguidas de letras diferentes, maiúscula na horizontal e minúscula na vertical, diferem entre si pelo teste Scott-Knott $(P<0,05)$. 
estudados o que se deve a fatores tais como: teor reduzido de matéria orgânica, à menor agregação, à menor penetração de raízes, ou ao maior adensamento ocasionado pelo peso das camadas sobrejacentes (Araújo et al. 2004).

Em relaçáo ao teor de argila houve diferença apenas entre os sistemas de uso. Os solos sob sistema MA, PA e T5 apresentaram os maiores teores de argila e menores densidades, seguidos dos sistemas agroflorestais T8, TC e TCP. No geral solos argilosos possuem menor densidade, principalmente pelo fato de possuírem maior porosidade total (Silva et al. 2006).

Os sistemas MA, PA e T5, além dos maiores teores de argila, possuem também elevados estoque de nutrientes. O aumento do teor de argila também está diretamente relacionado com o aumento do conteúdo de matéria orgânica no solo (Roscoe e Machado 2002), fato atribuído à maior quantidade de sítios de adsorção na superfície mineral das argilas por unidade de solo (Neufeldt et al. 2002).

Os resultados dos estoques de nutrientes, teores de argila e densidade referentes aos sistemas de uso, foram ordenados por meio da Análise de Componentes Principais- ACP, que relacionou as variáveis em estudo (Figura 1), onde os componentes principais 1 e 2 , explicaram $31,3 \%$ e $20,9 \%$ da variabilidade total dos dados, respectivamente. Os escores do componente principal 1 correlacionaram-se positivamente com o estoque de NT $(0,75)$ e $\mathrm{MO}(0,73)$, negativamente com o estoque de $\mathrm{Mg}(-0,50)$ e densidade $(-0,74)$. O componente principal 2 correlacionou-se negativamente com estoque de $\mathrm{K}(-0,79)$ e $\mathrm{Ca}(-0,68)$.
Assim, pode-se dizer que o componente principal 1 está relacionado à parte orgânica do solo, visto que sua correlação com a MO e o NT é elevada. Este por sua vez, é influenciado pela $\mathrm{MO}$, pois o conteúdo de matéria orgânica no solo é um bom índice de NT no solo, visto que a quase totalidade do nitrogênio se encontra na forma orgânica. A mineralizaçáo da matéria orgânica do solo, que engloba os processos de aminação e amonificação é responsável, por ano, pela conversão de 2 a $5 \%$ do $\mathrm{N}$ orgânico em $\mathrm{N}$ mineral. Esse processo é regulado pelo uso do solo (D'Andréa et al. 2004).

Valor de correlação significativo e positivo com o teor de MO já era esperado, em razão de se tratar de sistemas agroflorestais. Segundo Marin (2002), esses sistemas aumentam ou mantém os níveis de matéria orgânica do solo quando comparado com a mata nativa, devido ao maior aporte de resíduos que esses sistemas recebem ao longo dos anos.

Entre os atributos que se correlacionaram de maneira elevada e negativa com o segundo componente estão os estoques de $\mathrm{K}$ e $\mathrm{Ca}$. O Ca foi o elemento mais armazenado pelos sistemas de uso, diferente do K que foi o menos armazenado. $\mathrm{O}$ resultado das componentes principais permite confirmar que o Ca está adsorvido mais fortemente aos colóides do solo, nesses sistemas de uso, que o $\mathrm{K}$, dificultando assim, seu armazenamento (Ernani 2008).

Ainda observou-se que os teores de argila e o estoque de $\mathrm{P}$ não se correlacionaram com as outras variáveis estudadas, indicando que os sistemas de uso, não foram influenciados por estes. Diante destes resultados, os sistemas

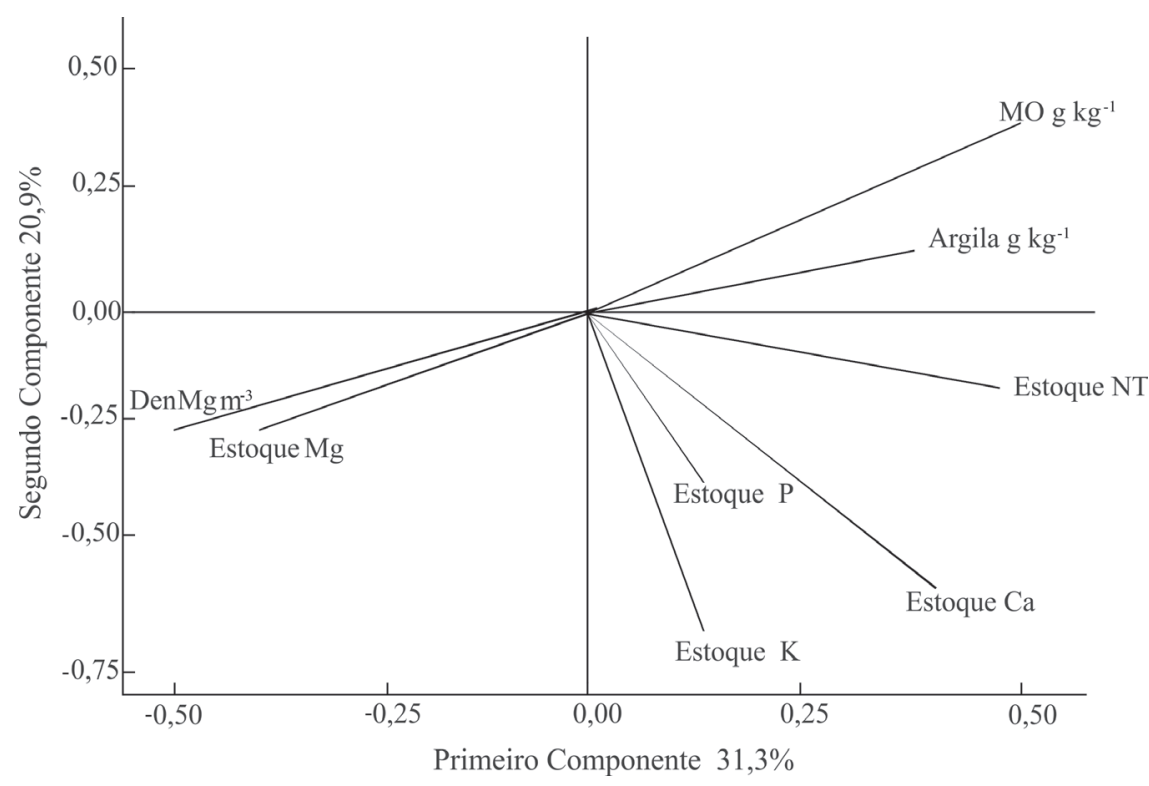

Figura 1 - Componentes principais (PCA-1 e PCA-2) dos resultados dos estoques de nutrientes em kg ha-1 (NT = Nitrogênio total, $\mathrm{P}=$ fósforo, $\mathrm{K}=$ potássio, $\mathrm{Ca}=$ Cálcio e $\mathrm{Mg}=$ Magnésio, matéria orgânica(M0), densidade (Den) e teores de argila em diferentes sistemas de uso. 
de uso apresentaram, de modo geral, altos teores de $\mathrm{MO}$ e armazenaram mais NT e Ca.

Com a análise de agrupamento dos estoques de nutrientes, densidade e argila, foi possível identificar grupos de sistemas de uso que se assemelham dentro de cada profundidade estudada (Figura 2).

$\mathrm{Na}$ profundidade de 0 a $5 \mathrm{~cm}$ foi possível identificar três grupos. O grupo 1 formado apenas pelo sistema MA; esse sistema de uso possui elevado estoque de NT e Ca e alto teor de MO nessa profundidade (Tabela 2), sendo assim formou um grupo isolado, não semelhante aos demais. O grupo 2 formado pelos sistemas T8, T5 e TC e grupo 3 pelos sistemas TCP e $\mathrm{PA}$, que apresentaram similaridade entre as variáveis avaliadas.

$\mathrm{O}$ sistema MA isolado em um grupo evidenciou que a prática agrícola causou alteração nos estoques de nutrientes e atributos físicos (argila e densidade) do solo quando estes, sob vegetação nativa, foram incorporados aos sistemas produtivos.

Souza et al. (2005) avaliaram atributos físicos e químicos em um Neossolo Quartzarênico, sob diferentes usos: pastagem, integraçáo lavoura-pecuária e Cerrado e observaram aumento na densidade do solo e reduçáo dos nutrientes nas áreas estudadas, quando comparadas com o Cerrado nativo.

Para as variáveis analisadas na camada de 5 a $10 \mathrm{~cm}$, observou-se que a influência do sistema de uso não alterou de maneira expressiva, os estoques de nutrientes, densidade e argila no T5, uma vez que esse sistema formou um grupo juntamente com o MA. Resultados semelhantes foram observados por Barreto et al. (2006) na avaliação da substituição da vegetação nativa de Mata Atlântica pelos sistemas agrícolas de produção de cacau e pastagem, em que as características físicas do solo não apresentaram diferença entre os usos agrícolas em comparação com a vegetação nativa.

Além do grupo formado pelos sistemas MA e T5, na profundidade de 5 a $10 \mathrm{~cm}$ houve a formação de mais dois grupos um formado pelos sistemas TC e PA, e outro por T8 e TCP, indicando que esses grupos foram submetidos à alteraçóes mais intensas, os diferenciando-os do sistema MA.

Na profundidade de 10 a $20 \mathrm{~cm}$ os sistemas MA, PA e T5 se agruparam e os sistemas T8, TCP e TC formaram o segundo grupo. Assim como na profundidade de 5 a 10 os sistemas MA e T5 formam o mesmo grupo, já os sistemas T8, TCP e TC continuaram não se assemelhando ao $\mathrm{MA}$, indicando que esses sistemas foram os mais influenciados pela conversáo da mata nativa em sistemas agrícolas, concordando com Perin et al. (2003) que observaram alteraçóes nos atributos químicos de solos incorporados a sistemas agrícolas.

$\mathrm{Na}$ camada de 20 a $30 \mathrm{~cm}$ não houve a formação de grupos indicando que a partir dessa profundidade não há diferença entre os sistemas de uso quanto às variáveis analisadas. Assim, as alteraçóes provocadas pelo sistema de uso do solo se restrigem apenas as camadas de 0 a 5,5 a 10 e 10 a $20 \mathrm{~cm}$, o que também foi observado por Côrrea et al. (2010).
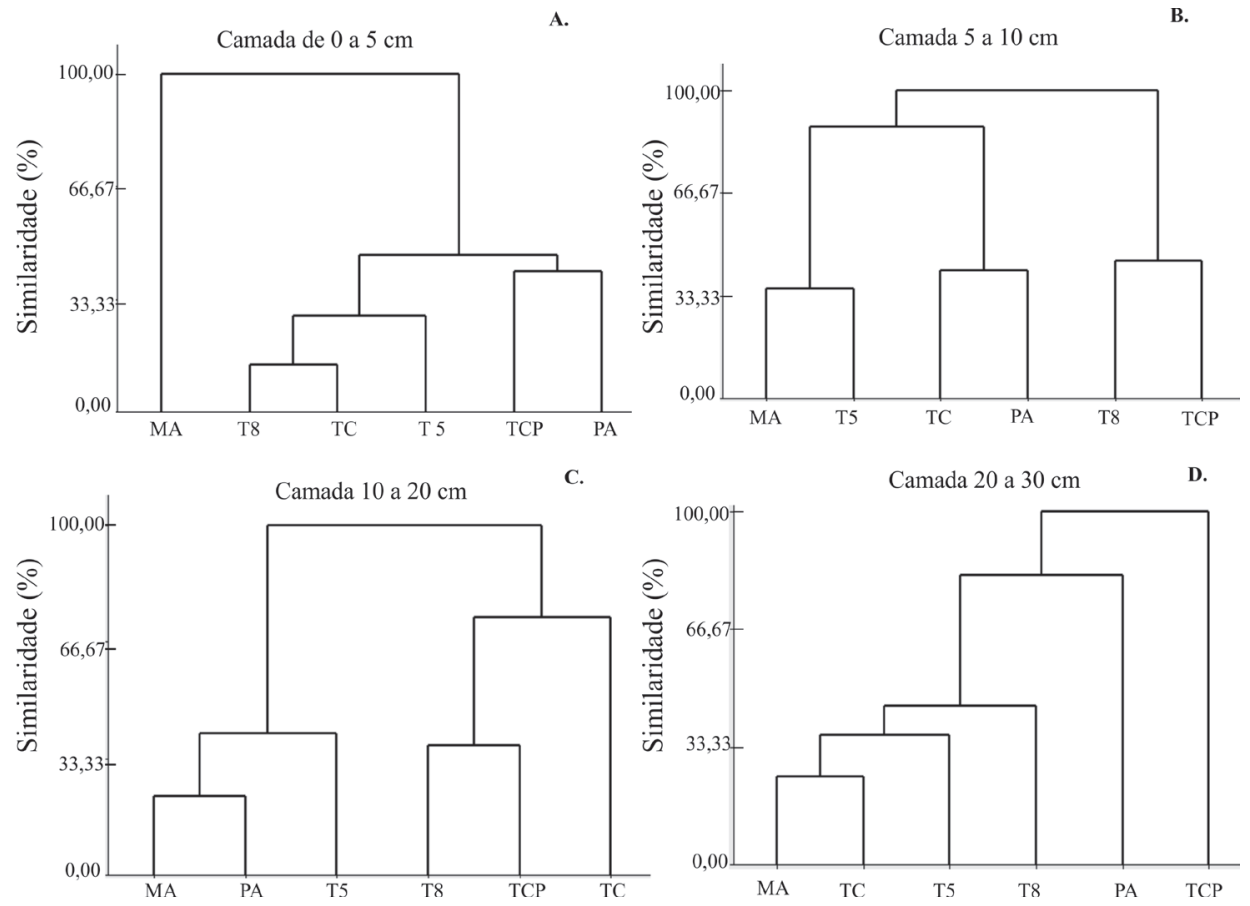

Figura 2 - Agrupamento hierárquico dos sistemas de uso por semelhança dos estoques, densidade e teor de argila, dentro de cada camada estudada. MA= mata, $\mathrm{T} 8=$ teca 8 anos, $\mathrm{T} 5=$ teca 5 anos, $\mathrm{TCP}=$ teca, cacau e pasto, $\mathrm{TC}=$ teca e cacau, $\mathrm{PA}=$ pasto. 


\section{CONCLUSÕES}

1. Os estoques de nutrientes alteram quando a vegetação nativa é incorporada aos sistemas produtivos.

2. O nutriente mais estocado em todos os sistemas de uso é o Ca, os valores variaram entre 5188,48 e $2912,86 \mathrm{~kg} \mathrm{ha}^{-1}$ e o menos estocado o $\mathrm{K}$, com estoques entre 46,22 e 17,33 $\mathrm{kg} \mathrm{ha}^{-1}$.

3. A análise de componentes principais reduz as variáveis originais para dois componentes principais que representam $52,2 \%$ da variação total dos dados, sendo que as variáveis que mais se destacam são $\mathrm{MO}$, e os estoques de $\mathrm{N}$ e Ca.

4. O sistema teca com cinco anos foi o sistema que mais se aproximou da mata, quanto ao armazenamento de nutrientes

\section{AGRADECIMENTOS}

À Coordenação de Aperfeiçoamento de Pessoal de Nível Superior e ao Programa de Pós-graduação em Agricultura Tropical da Universidade Federal de Mato Grosso.

\section{BIBLIOGRAFIA CITADA}

Altieri, M. 2002. Agroecologia: bases científicas para uma agricultura sustentável. Agropecuária, Guaíba-RS. 592 pp.

Araújo, E. A.; Ker, J. C, Mendonça, E. S.; Silva, I. R.;Oliveira, E.K. 2011. Impacto da conversão floresta - pastagem nos estoques e na dinâmica do carbono e substâncias húmicas do solo no bioma Amazônico. Acta Amazonica, 41:103-114.

Araújo, E. A.; Lani, J.L. ; Amaral, E.F.; Guerra, A. 2004. Uso da terra e propriedades físicas e químicas de Argissolo Amarelo distrófico na Amazônia Ocidental. Revista Brasileira de Ciência do solo, 28: 307-315.

Araújo, Q.K.; Figueiredo, M.S.; Costa, L.M.; Loures, E.G.; Regazzi, A.J.; Fontes, L.E.F.; Casali, V.W.D. 1994. Ação da queima e da percolação sobre propriedades químicas de um Latossolo Vermelho-Amarelo variação una. Revista Ceres, 41:537-558.

Barreto, A. C.; Lima, F. H. S.; Freire, M. B. G. S.; Araújo, Q. R.; Freire, F. J. 2006. Características químicas e físicas de um solo sob floresta, sistema agroflorestal e pastagem no Sul da Bahia. Caatinga, 19:415-425.

Bayer, C.; Mielniczuk, J.; Amado, T. J. C.; Martin-Neto, L.; Fernandes, S. A.2000. Organic matter storage in a sandy loam Acrisol affected by tillage and cropping systems in southern Brazil. Soil and Tillage Research, 54: 101-109.

Bremner, J. M, Mulvaney, C.S.1982. Total nitrogen, p.595-624. In:PAGE, A. L. Methods of soil analysis. American Society of Agronomy, Madson.

Conceição, M.; Manzatto, C. V.; Araújo, W.S.;Matin Neto, L.; Saab, S. C.;Cunha, T. J. F.; Freixo, A.A. 1999. Pesquisa em Andamento $\mathrm{n}^{\circ}$ 03, Embrapa-Solos.p 1-5.
Corrêa, R. M.; Freire, M. B. G. dos S.; Ferreira, R. L. C.; Silva, J. A. A. da; Pessoa, L. G. M.; Miranda, M. A.; Melo, D. V. M. 2010. Atributos físicos de solos sob diferentes usos com irrigação no semiárido de Pernambuco. Revista Brasileira de Engenharia Agricola e Ambiental, 14: 358-365.

Costa, F. de S.; Bayer, C.; Albuquerque, J. A.; Fontoura, S. M. 2004. Aumento da matéria orgânica num Latossolo Bruno em plantio direto. Ciência Rural, 34: 587-589.

D’Andréa, A.F.; Silva, M.L.N.; Curi, N.; Guilherme, L.R.G. 2004. Estoque de carbono e nitrogênio e formas de nitrogênio mineral em solo submetido a diferentes sistemas de manejo. Pesquisa Agropecuária Brasileira, 39:179-186.

Ellert, B. H.; Bettany, J. R.1995. Calculation of organic matter and nutrients stored in soils under contrasting management regimes. Canadian Journal Soil Science, 75:529-538.

EMBRAPA. Centro Nacional de Pesquisa de Solos. 1997. Manual de métodos de análise de solos. 2.ed. Rio de Janeiro, Rio de Janeiro. 212pp.

Ernani, P.R.2008. Química do solo e disponibilidade de nutrientes. O Autor, Lages. 230 pp.

Falleiro, R.M.; Souza, C.M;Silva, C.S.W.;Sediyama,C.S;Silva, A.A.; Fagundes, J.L. 2003. Influência dos sistemas de preparo nas propriedades químicas e físicas do solo. Revista Brasileira de Ciência do solo, 27:1907-1104.

Galvão, J. R.; Morais, F. I. O de.;Tofoli, R. de C. Z.2009. Massa seca e limitaçóes nutricionais do milho em um Latossolo Amarelo, sob floresta secundária e sistemas agroflorestais. Revista de Ciência Agrária, 52:137-145.

Governo do Estado De Rondônia/Seplan - RO.2002. Plano estratégico de desenvolvimento do Estado de Rondônia. Seplan, Porto Velho.

Governo do Estado De Rondônia/Sedam - RO. 2010. Acervo Técnico de Zoneamento (http://www.sedam.ro.gov.br/index. php/acervo-tecnico-zoneamento.html). Acesso em 13/03/012.

Hair Jr., J. F.; Anderson, R. E.; Tatham, R. L. ; Black, W. C.2005. Análise multivariada de dados. Porto Alegre, Bookman.593 pp.

Johnson, R.A.; Wichern, D.W.1998. Applied multivariate statistical analysis. New Jersey, Prentice Hall. 816 pp.

Lima, S. S. L.; Leite, L. F. C.;Oliveira, F. C.; Costa, D. B. 2011. Atributos químicos e estoques de carbono e nitrogênio em argissolo vermelho-amarelo sob sistemas agroflorestais e agricultura de corte e queima no norte do Piauí. Revista Árvore, 35:51-60.

Longo, R.M.; Espíndola, C.R. 2000. Alteraçôes em características químicas de solos da região Amazônica pela introdução de pastagens. Acta Amazonica, 30: 71-80.

Marin, A.M.P. 2002. Impactos de um sistema agroflorestal com café na qualidade do solo. Dissertação de Mestrado. Universidade Federal de Viçosa. Viçosa, Minas Gerais. 83 pp. 
Marques, J. D. de O.; Teixeira, W. G.; Reis, A. M.; Cruz Junior, O. F.; Batista, M. S.; Branco Afonso, M.A.C. 2010. Atributos químicos, físico-hídricos e mineralogia da fração argila em solos do Baixo Amazonas: Serra de Parintins. Acta Amazonica.40: 01-12.

Martinez, L. J. ; Zinck, J. A. 2004. Temporal variation of soil compaction and deterioration of soil quality in pasture areas of Colombian Amazônia. Soil and Tillage Research, 75: 3-17.

McGrath, D.A.; Duryea, M.L.; Cropper, W.P. 2001. Soil phosphorus availability and fine root proliferation in Amazonian agroforests 6 years following Forest conversion. Agriculture, Ecosystems and Environment ,83:271-284.

Mokry, M. 2003. Makroporen Transport Von Phosphor Gulleausbringung auf Loss-und Tonboden. (http:/www. gumpenstein.at/publikationen/lysimeter2003/mokry.pdf -.---). Acesso em 20/08/2009.

Moreira, A.; Malavolta, E. 2004. Dinâmica da matéria orgânica e da biomassa microbiana em solo submetido a diferentes sistemas de manejo na Amazônia Ocidental. Pesquisa Agropecuária Brasileira, 39:1103-1110.

Moreira, A.; Costa, D. G. 2004. Dinâmica da matéria orgânica na recuperação de clareiras da floresta amazônica. Pesquisa Agropecuária Brasileira. 39: 1013-1019.

Neufeldt,H.; Resck, D. V. S.; Ayarza, M. A.; Zech. W. 2002. Texture and land -use effects on soil organic matter um Cerrado oxisols, Central Brazil. Geoderma,107:151-164.

Perin, E.; Ceretta, C.A.; Klamt, E. 2003. Tempo de uso agrícola e propriedades químicas de dois Latossolos do planalto médio do Rio Grande do Sul. Revista Brasileira Ciência do Solo, 27:665-674.

Rangel, O.J.P. ; Silva, C.A.2007. Estoques de carbono e nitrogênio e fraçôes orgânicas de Latossolo submetido a diferentes sistemas de uso e manejo. Revista Brasileira de Ciência do Solo, 31:16091623.
Roscoe, R.; Machado, P. L. O. D. A. 2002. Fracionamento Físico do Solo em Estudos da Matéria Orgânica. Dourados, Rio de Janeiro. 86 pp.

Silva, R. C.; Pereira, J. M.; Araújo, Q. R.; Pires, A. J. V.; Rei, A.J. 2007. Alterações nas propriedades químicas e físicas de um Chernossolo com diferentes coberturas vegetais. Revista Brasileira de Ciência do solo, 31:101-107.

Silva, M. A. S.; Mafra, A. L.; Albuquerque, J. A.; Rosa, J. D.; Bayer, C.; Mielniczuk , J.2006. Propriedades físicas e teor de carbono orgânico de um Argissolo Vermelho sob distintos sistemas de uso e manejo. Revista Brasileira de Ciência do Solo, 30 : 329-337.

Silva, I.R. da; Novais, R.F. de; Barros, N.F. de; Silva, E. F.2004. Manejo de resíduos e matéria orgânica do solo em plantaçóes de eucalipto: uma questáo estratégica para a manutenção da sustentabilidade. Revista Brasileira de Ciência do Solo, 29:10-20.

Silva, S.C.1995. Condiçôes edafo-climáticas para a produção de Panicum sp. p.147-196. In: Simpósio Sobre Manejo De Pastagem,12. Piracicaba. Anais.Piracicaba, Escola Superior de Agricultura Luiz de Queiroz, 1995.

Souza, E. D.; Carneiro, M. A. C.; Paulino, H. B.2005. Atributos físicos de um Neossolo Quartzarênico e um Latossolo Vermelho sob diferentes sistemas de manejo. Pesquisa Agropecuária Brasileira, 40:1135-1139.

Souza, Z.M.; Alves, M.C. 2003. Propriedades físicas e teor de matéria orgânica em um Latossolo Vermelho de Cerrado sob diferentes usos e manejos. Acta Scientiarum: Agronomy. 25:27-34

Swarowsky,A.;Righes, A. A.; Marchezan, E.;Rhoden, A. C.; Guniani, E.I. 2006. Concentração de nutrientes na solução do solo, sob diferentes manejos do arroz irrigado. Revista Brasileira de Engenharia Agricola e Ambiental. 10: 344-351.

Recebido em: 25-10-2011

Aceito em:28-03-2012 